\title{
NEW EXCITATIONS IN THE THIRRING MODEL
}

\author{
J.L. Cortés ${ }^{1 *}$ J. Gamboa ${ }^{2 \dagger}$, I. Schmidt ${ }^{3 \ddagger}$ and J. Zanelli ${ }^{2,4} \S$ \\ ${ }^{1}$ Departamento de Fúsica Teórica, Universidad de Zaragoza, Zaragoza 50009, Spain \\ ${ }^{2}$ Departamento de Física, Universidad de Santiago de Chile, Casilla 307, Santiago 2, Chile \\ ${ }^{3}$ Departamento de Física, Universidad Técnica F. Santa Maria, Valparaíso, Chile \\ ${ }^{4}$ Centro de Estudios Científicos de Santiago, Casilla 16443, Santiago, Chile
}

The quantization of the massless Thirring model in the light-cone using functional methods is considered. The need to compactify the coordinate $x^{-}$in the light-cone spacetime implies that the quantum effective action for left-handed fermions contains excitations similar to abelian instantons produced by composite of left-handed fermions. Righthanded fermions don't have a similar effective action. Thus, quantum mechanically, chiral symmetry must be broken as a result of the topological excitations. The conserved charge associated to the topological states is quantized. Different cases with only fermionic excitations or bosonic excitations or both can occur depending on the boundary conditions and the value of the coupling.

In recent years quantization in the light cone frame has been extensively studied in connection with the discovery of new non-perturbative effects that would be unobservable in the standard spacetime quantization [1] 3].

There are several reasons that make the light cone quantization a method radically different compared to the standard quantization. The dispersion relation $k_{\mu} k^{\mu}=m^{2}$ becomes $k^{+} k^{-}=m^{2}$, in the light cone. This implies that the particles and antiparticles occupy disconnected sectors of momentum space. Furthermore, if the momentum $k^{+}$is discretized by compactifying $x^{-}$, the energy $k^{-}$is also quantized and nonzero. Thus, the light cone momentum is never singular if the spacetime topology is $S^{1} \times \Re$ 细.

The above comments show that the quantization in the light cone is naturally defined over a manifold with non-trivial topology. As a consequence, one could expect new physical effects originated in the implicit difference in topology from standard spacetime quantization.

From this point of view, the massless Thirring model is an example where one could investigate the new effects that emerge in the light-cone frame. We will show below that, contrary to the standard quantization, the non-trivial topology of the light-cone spacetime induces abelian instanton-like excitations, i.e. a purely quantum

\footnotetext{
${ }^{*}$ E-mail: cortes@leo.unizar.es

${ }^{\dagger}$ E-mail: jgamboa@lauca.usach.cl

${ }^{\ddagger}$ E-mail: ischmidt@fis.utfsm.cl

§E-mail: jz@cecs.cl
}

mechanical effect that appears in the calculation of the fermionic determinant.

Let us consider the massless Thirring model in the light cone frame

$$
\mathcal{L}=i \psi^{\dagger}{ }_{L} \partial_{+} \psi_{L}+i \psi^{\dagger}{ }_{R} \partial_{-} \psi_{R}-2 g^{2}\left(\psi^{\dagger}{ }_{L} \psi_{L}\right)\left(\psi^{\dagger}{ }_{R} \psi_{R}\right),
$$

where $\partial_{ \pm}=\frac{\partial}{\partial x^{ \pm}}$and $x^{+}$and $x^{-}$play the role of time and space respectively.

One should note that (11) is invariant under the charge conjugation symmetry $\psi_{L, R} \leftrightarrow \psi_{L, R}^{\dagger}$, that is expected to be conserved at the quantum level. However in order to quantize the system following the path integral methods it is more convenient to write (Ii) as follows

$$
\begin{aligned}
\mathcal{L} & =\psi_{R}^{\dagger}\left(i \partial_{-}-2 g^{2} \psi^{\dagger}{ }_{L} \psi_{L}\right) \psi_{R}+\psi^{\dagger}{ }_{L}\left(i \partial_{+}\right) \psi_{L} \\
& =\psi_{R}^{\dagger}\left(i \partial_{-}+2 A_{-}\right) \psi_{R}+\psi_{L}^{\dagger}\left(i \partial_{+}\right) \psi_{L}
\end{aligned}
$$

where $A_{-}=-g^{2} \psi_{L}^{\dagger} \psi_{L}$.

The symmetry under charge conjugation including the auxiliary field $A_{-}$is now

$$
\psi_{L}^{\dagger} \leftrightarrow \psi_{L}, \quad \psi_{R}^{\dagger} \leftrightarrow \psi_{R}, \quad A_{-} \rightarrow-A_{-} .
$$

Thus, the generating functional,

$$
Z=\int \mathcal{D} \psi_{R}^{\dagger} \mathcal{D} \psi_{R} \mathcal{D} \psi_{L}^{\dagger} \mathcal{D} \psi_{L} e^{i S},
$$

after integrating over the right handed fields is

$$
Z=\int \mathcal{D} \psi_{L}^{\dagger} \mathcal{D} \psi_{L} \operatorname{det}\left(i \partial_{-}+2 A_{-}\right) e^{i \int d x^{+} d x^{-} \psi_{L}^{\dagger} i \partial_{+} \psi_{L}},
$$

where $i \partial_{-}+2 A_{-}$is a one-dimensional Dirac operator. It should be noted that $A_{-}=A_{-}\left(x^{-}, x^{+}\right)$is a function of two variables. The eigenvalues $\lambda_{n}$ of the equation

$$
\left[i \partial_{-}+2 A_{-}\right] \varphi_{n}=\lambda_{n} \varphi_{n}
$$

are parametric functions of $x^{+}$, which can be determined integrating (6) with $x^{+}$fixed.

On the other hand, as $i \partial_{-}+2 A_{-}$describes a fermionic system, usual practice would be to solve (5) using antiperiodic boundary conditions. However, the periodic topology of $x^{-}$also allows for twisted boundary conditions 


$$
\psi\left(x^{+}, \sigma\right)=e^{2 \pi i \gamma\left(x^{+}\right)} \psi\left(x^{+}, 0\right),
$$

where the real parameter $\gamma$ should be fixed by quantum consistency.

The solution of (6) is

$$
\varphi_{n}\left(x^{+}, x^{-}\right)=e^{i \int_{0}^{x^{-}} d y\left[2 A_{-}\left(x^{+}, y\right)-\lambda_{n}\right]} \chi_{0},
$$

where $\chi_{0}$ is a Grassmann spinor independent of $x^{-}$. Using (7) the eigenvalues are found to be

$$
\lambda_{n}=-\frac{2 \pi(\gamma+n)}{\sigma}+\frac{2}{\sigma} \int_{0}^{\sigma} d x^{-} A_{-} .
$$

Using $\zeta$-function regularization, the determinant is found to be

$$
\Gamma_{\gamma}(A)=\operatorname{det}\left(i \partial_{-}+2 A_{-}\right)=\mathcal{N} \sin (\Phi-\pi \gamma),
$$

where $\Phi=\int_{0}^{\sigma} d x^{-} A_{-}$is the "magnetic flux" produced along the surface $x^{+}=$const. and $\mathcal{N}$ is a normalization constant.

Thus, the effective quantum theory for the left handed fields reads

$$
Z=\int \prod_{x^{+}} \mathcal{D} \psi_{L}^{\dagger} \mathcal{D} \psi_{L} \Gamma_{\gamma}(A) e^{i \int d x^{+} d x^{-} \psi_{L}^{\dagger}\left(i \partial_{+}\right) \psi_{L}},
$$

with $\Gamma_{\gamma}(A)$ given by $(10)$.

In order to preserve quantum mechanically the charge conjugation symmetry, the boundary conditions have to be consistent with the transformation $\psi^{\dagger} \leftrightarrow \psi$. This requires that

$$
e^{2 \pi i \gamma}=e^{-2 \pi i \gamma}
$$

and then $\gamma$ must be an integer (periodic boundary conditions) or a half-integer (antiperiodic boundary conditions). The next step is to observe that the boundary condition (7) is invariant under the shift $\gamma \rightarrow \gamma+1$. This implies

$$
\Gamma_{\gamma}(A)=\Gamma_{\gamma+1}(A)
$$

which requires $\sin (\Phi-\pi \gamma)=\sin (\Phi-\pi(\gamma+1))$, or equivalently

$$
\sin (\Phi-\pi \gamma)=0
$$

In view of the assertion (12), the only consistent solutions of (14) are:

$\Phi=\left(m+\frac{1}{2}\right) \pi, \quad$ and $\quad \gamma=m^{\prime}+\frac{1}{2}, \quad$ with $\quad m, m^{\prime} \in \mathbf{Z}$,

\footnotetext{
${ }^{1}$ Note that, although $A_{-}$is bilinear in $\psi$, any power of the integral on $A_{-}$is different from zero; then equation (9) is well defined.
}

or,

$$
\Phi=m \pi, \text { and } \gamma=m^{\prime}, \text { with } m, m^{\prime} \in \mathbf{Z} .
$$

In summary, the Thirring fields obey either antiperiodic boundary conditions with half-integer flux, or periodic boundary conditions with integer flux.

As a consequence of (14) the fermionic determinant satisfies the condition

$$
\Gamma_{\gamma}(A)=\Gamma_{-\gamma}(-A)
$$

and the theory is invariant under charge conjugation. In fact, condition (14) means that the generating function for the effective theory vanishes. This should come as no surprise because, as noted by 't Hooft, the path integral for a massless fermion vanishes when the Fermi field couples to a gauge field with nontrivial topology [5] (see also [6]).

This result shows that there are two points of view to analyze this problem. Before integrating out $\psi_{R}$ each Fermi field interacts with a background made out of fermions of the opposite chirality. After integrating out one of the two species, the remaining field self interacts with its own condensate $\psi^{\dagger} \psi$. In our discussion $\psi_{L}^{\dagger} \psi_{L}$ plays the role of an external background gauge field $A_{-}$ with non-trivial topology interacting with the $\psi_{L}^{\dagger}$. As a consequence of the light-cone quantization, the left-right symmetry is broken because $x^{-}$is compactified but not $x^{+}$. Thus, although the starting classical action (1) is symmetric under $\psi_{L} \leftrightarrow \psi_{R}$, quantum mechanically only left-handed charge is conserved.

In fact, the conserved Noether charge associated with a rigid phase rotation of the effective action (11) is

$$
Q_{-}=: \int_{0}^{\sigma} d x^{-} \psi_{L}^{\dagger} \psi_{L}
$$

while a similar conserved charge for the right-handed fermions is not defined in the effective theory. Furthermore, this charge is quantized by virtue of (15) or (16). In the previous form of the path integral (4), the righthanded charge $Q_{+}=: \int_{0}^{\sigma} d x^{-} \psi_{R}^{\dagger} \psi_{R}$, is classically conserved but obeys an anomalous quantum algebra with $Q_{-}$.

At this point one could note that the expression for the determinant of the one-dimensional Dirac operator obtained by using $\zeta$-function is not the most general expression for the regularized determinant. The general form of the regularized one-dimensional determinant is

$$
\Gamma_{\gamma}(A)=e^{a+b \xi} \sin \xi,
$$

where $\xi=\Phi-\pi \gamma$ and $a$ and $b$ are arbitrary coefficients that depend on the regularization procedure. This general form is obtained starting from the formal expression of $\Gamma_{\gamma}(A)$ as an infinite product of eigenvalues depending on $\xi$. In order to give meaning to this product one considers the logarithm as a (divergent) sum and takes 
enough derivatives with respect to $\xi$ (two) until a convergent sum, which is the series representation of $\sin ^{-2} \xi$, is obtained. The linear expression $a+b \xi$ gives the most general $\xi$-dependence due to the divergence of the onedimensional determinant. The result of $\zeta$-function regularization corresponds to the particular choice of the regularization parameter $b=0$ and $e^{a}$ is the normalization constant $\mathcal{N}$ in (10).

If one takes the general form (19) for the regularized one-dimensional determinant, the consistency condition (13) leads now to

$$
\left[1+e^{-b \pi}\right] \sin (\Phi-\pi \gamma)=0
$$

instead of (14), and this implies once more a quantization of the flux, (15) or $(16)$, for $b \neq i(2 n+1)$. The possibility to have a charge conjugation invariant theory without a quantized flux by choosing appropiately the regularization, $b=i(2 n+1)$, deserves further investigation.

We end up this discussion by pointing out that an important information on the non-perturbative spectrum of the model can be read from the relation, $\Phi=-g^{2} Q_{-}$, between the quantized flux $\Phi$ and the conserved charge $Q_{-}$which counts the number $n_{L}$ of left-handed fermions in a fixed $-x^{+}$surface.

In the case of periodic boundary conditions $m \pi=$ $-g^{2} n_{L}$; then one has $m=n_{L}=0$, i.e. only neutral bosonic $2^{2}$ excitations, unless $g^{2} / \pi$ is a rational number. If $g^{2} / \pi=p / q$ then the quiral charge has to be a multiple of $q$ and the magnetic flux will be $\Phi=-\pi n_{L} p / q$. For $q$ even one has bosonic excitations while for $q$ odd the spectrum contains both bosonic and fermionic excitations.

In the case of antiperiodic boundary conditions one has $(m+1 / 2) \pi=-g^{2} n_{L}$ and $g^{2} / \pi$ has to be a rational number with even denominator. Only states, characterized by an integer $n$, with a quiral charge $n_{L}=(2 n+1) q / 2$ and a flux $\Phi=-(n+1 / 2) p \pi$ appear in the spectrum; for $q / 2$ even one has a purely bosonic spectrum but in the case $q / 2$ odd only fermionic excitations are present.

The results presented here can be summarized as follows: (i) A fermionic topological instanton-like excitation arising from the compactification of the $x^{-}$; (ii) Quantum mechanically, the massless Thirring model is analogous to the $\lambda \phi^{4}$ theory in $1+1$ dimensions, where an abelian instanton is also possible (see e.g. 7); (iii) different situations with only fermionic excitations, bosonic excitations or both can be identified ; (iv) the quantum system breaks the classical left-right symmetry.

The lesson we've learned is that a simple self-interacting fermionic system can have many more excitations than

\footnotetext{
${ }^{2}$ We use the term "Bosons" for excitations which are symmetric under particle exchange, even though the path integral is calculated using Grassmann fields.
}

those expected perturbatively. This last statement is particularly interesting for $\mathrm{QCD}_{4}$ at low energies where this kind of theory - v.i.z. the Nambu-Jona-Lasinio modelis expected. It remains to be proven whether a similar construction can be carried out in higher dimensions.

Acknowledgements We thank M. Asorey, J. Beckers, M. Henneaux and M. Tytgat for useful discussions and hospitality in Zaragoza, Liege and Brussels. This work was partially supported by CICYT (Spain) project AEN97-1680 and grants 1980788, 1960229, 19605367960001 from FONDECYT-Chile, and DICYT-USACH. I.S. is a recipient of a Cátedra Presidencial en Ciencias-Chile. Institutional support to CECS from Fuerza Aerea de Chile and a group of private companies (Business Design Associates, CGE, Codelco, Copec, Empresas CMPC, Minera Colahuasi, Minera Escondida, Novagas, and XeroxChile), is also acknowledged. J.Z is a J.S. Guggenheim Memorial Foundation fellow.

[1] S. Brodsky, H.-C. Pauli and S. Pinsky, hep-th/9705477, Phys. Rep. (in press).

[2] M. M. Brisudova, R. Perry and K.G. Wilson, Phys. Rev. Lett. 78, 1227 (1997); S. D. Glazek and K. G. Wilson, Phys. Rev. D57, 3558 (1998).

[3] D. Bigatti and L. Susskind, hep-th/9711063.

[4] T. Maskawa and K. Yamawaki, Prog. Theor. Phys. 56270 (1976); S. Brodsky and H. -C. Pauli, Phys. Rev.32, 1993 (1985).

[5] G. 't Hooft, Phys. Rev. Lett.37, 8 (1976); i.b.i.d. Phys. Rev.D14, 3432 (1976).

[6] J. Kiskis, Phys. Rev.15D, 2329 (1977).

[7] S. Coleman, Aspects of Symmetry, Cambridge University Press; A. M. Polyakov, Gauge Fields and Strings, Harwood (1988). 